\title{
Predicting DNAPL entrapment and recovery: the influence of hydraulic property correlation
}

\author{
L. D. Lemke, L. M. Abriola
}

\begin{abstract}
The influence of aquifer property correlation on multiphase fluid migration, entrapment and recovery was explored by incorporating correlated and uncorrelated porosity, permeability, and capillary pressure-saturation $\left(\mathrm{P}_{\mathrm{c}^{-}}\right.$ Sat) parameter fields in a cross-sectional numerical multiphase flow model. Comparison of two-dimensional entrapped organic saturation distributions for a simulated tetrachloroethylene (PCE) spill in ensembles of aquifer realizations suggests that the degree of spatial correlation in $\mathrm{P}_{\mathrm{c}}$-Sat parameters exerts a controlling influence on dense nonaqueous phase liquid (DNAPL) spreading and redistribution in saturated aquifers. The predicted evolution of DNAPL source zones and resultant remediation efficiency under surfactant enhanced aquifer remediation (SEAR) also appear to be strongly influenced by the spatial correlation of aquifer parameters and multiphase flow constitutive relationships. Results for a limited number of realizations selected from each ensemble showed that removal of $60 \%$ to $99 \%$ of entrapped PCE could reduce dissolved contaminant concentration and mass flux by approximately two orders of magnitude under natural gradient conditions. Aqueous phase contaminant mass flux did not vary uniformly as a function of \% DNAPL removed, however, and notable differences in behavior were observed for models incorporating correlated versus uncorrelated $\mathrm{P}_{\mathrm{c}}$-Sat and permeability fields. Although these results must be confirmed through analysis of additional realizations, it is likely that similar or larger differences between correlated and uncorrelated system behavior will be observed in aquifers with greater spatially variability than that of the nonuniform, homogeneous sand aquifer studied here.
\end{abstract}

Keywords: Heterogeneity, DNAPL, DNAPL source zones, Surfactant enhanced aquifer remediation, SEAR

L. D. Lemke ( $\square)$, L. M. Abriola

University of Michigan, Department of Civil and Environmental

Engineering, Ann Arbor, Michigan, USA

e-mail: ldlemke@umich.edu

Funding for this research was provided by the United States Environmental Protection Agency, Great Lakes and Mid-Atlantic Center for Hazardous Substance Research under Grant No. R-825540, the Michigan Department of Environmental Quality under Contract No. Y80011, and the Strategic Environmental Research and Development Program under Project No. CU-1293. The content of this publication does not necessarily represent the views of these agencies and has not been subject to agency review. 


\section{Introduction}

Application of multiphase flow models for the prediction of dense nonaqueous phase liquid (DNAPL) migration and recovery requires estimation of the spatial distribution of aquifer properties such as intrinsic permeability, $k$, and porosity, $\phi$, as well as nonlinear constitutive relationships for capillary pressure-saturation $\left(\mathrm{P}_{\mathrm{c}}-\mathrm{Sat}\right)$, and relative permeability. Geostatistics is commonly used to model the spatial variability of permeability while uniform porosity is typically assumed. Capillary retention characteristics at each node of the geostatistical realization are then estimated by Leverett (1941) scaling of a representative capillary pressure curve. A consequence of this approach is the adoption of completely correlated $k$ and $\mathrm{P}_{\mathrm{c}}$-Sat distributions. This paper describes initial results of a numerical investigation designed to explore the influence of aquifer property correlation on predictions of multiphase fluid migration, entrapment, and recovery in an aquifer with statistically homogeneous but nonuniform physical properties.

The modeled aquifer is located in Oscoda, Michigan, USA, at the site of a former dry cleaning business. Aquifer characterization efforts were conducted in support of a Surfactant Enhanced Aquifer Remediation (SEAR) pilot scale test, designed to solubilize and recover residual tetrachloroethylene (PCE) from a suspected DNAPL source zone at the site. As part of the SEAR design effort, alternative spatial variability models of the unconfined aquifer were used to predict surfactant transport flow paths, travel times, and concentrations within the intended surfactant sweep zone (Drummond et al., 2000). Entrapped PCE distributions were also simulated for both a homogeneous and a spatially variable vertical cross-section using the immiscible fluid flow model MVALOR (Abriola et al., 1992; Rathfelder and Abriola, 1998). A uniform effective $k$ value was assigned to the homogeneous profile. Permeability values for the spatially variable profile were extracted from a three-dimensional, nonuniform, Gaussian geostatistical $k$ realization. Subsequent SEAR flushing of the two profiles was modeled using MISER (Abriola et al., 1997), a two-dimensional compositional simulator that can account for rate limited PCE dissolution and surfactant sorption. These initial simulations demonstrated that (a) small-scale nonuniformities within a single depositional facies have an important influence on the infiltration and redistribution of DNAPLs within such units, and (b) variability in the organic distribution within a DNAPL source zone directly influences predictions of DNAPL recovery (Abriola et al., 2001). This study extends the previous analysis through a more systematic investigation of the influence of aquifer property spatial variability models on predictions of DNAPL entrapment and recovery at this site.

\section{2}

\section{Methodology}

Porosity and grain size distribution data collected at 15 to $30 \mathrm{~cm}$ intervals from 12 vertical and directional cores were used to estimate hydraulic conductivity and capillary pressure-saturation curves (Lemke et al., 2000). Isotropic hydraulic conductivity, $K$, values were estimated from grain size distributions at 167 sample points using the Kozeny-Carman equation (Bear, 1972):

$K=\frac{\rho_{f} g}{\mu} k=\frac{\rho_{f} g}{\mu}\left[\frac{d_{m}^{2}}{180} \frac{\phi^{3}}{(1-\phi)^{2}}\right]$, 
where $\rho_{f}$ is the fluid (water) density, $\mathrm{g}$ is acceleration due to gravity, $\mu$ is the fluid dynamic viscosity, $k$ is the intrinsic permeability, $\phi$ is porosity, and $d_{m}$ is a representative grain size. The $d_{10}$ value was selected as a representative grain size value based on a minimization of error between Kozeny-Carman estimates and ten laboratory $K$ measurements. Assuming constant water density and viscosity, values of $k$ and $K$ are directly scaleable using Eq. (1). Although continuous core samples collected from the site contain relatively homogeneous fine to medium grained sand, estimated $k$ values were non-uniform, ranging from $1.21 \times 10^{-8}$ to $72.7 \times 10^{-8} \mathrm{~cm}^{2}$. The variance of $\ln (\mathrm{k})$ was 0.29 . Vertical and horizontal correlation lengths were 0.36 and $2.33 \mathrm{~m}$, respectively. These values are similar to those observed at the Canadian Forces Base Borden, Ontario site by Woodbury and Sudicky (1991). In addition, six soil classes were identified based on KMEANS clustering of the 167 measured grain size distributions following the approach of Schad (1993) (see Lemke et al., 2002, accepted for publication).

Capillary pressure-saturation relationships were modeled using a BrooksCorey (Brooks and Corey, 1964) formulation:

$\bar{S}_{w}=\left(\frac{P_{b}}{P_{c}}\right)^{\lambda}$ for $P_{c} \geq P_{b} ; \quad$ and $\quad \bar{S}_{w}=1.0$ for $P_{c}<P_{b}$,

where $P_{b}$ is the entry or bubbling pressure, $\lambda$ is the pore size distribution index, and $\bar{S}_{w}$, is the effective wetting phase saturation, defined as:

$\bar{S}_{w}=\frac{S_{w}-S_{w r}}{1-S_{w r}}$.

Here $S_{w r}$ is the residual or irreducible wetting phase saturation.

For primary drainage, both $P_{b}$ and $\lambda$ were estimated directly from grain size distribution curves and porosity values using the Haverkamp and Parlange (1986) method. Laboratory measured $\mathrm{P}_{\mathrm{c}}$-sat curves made using a pressure cell apparatus developed by Salehzadeh and Demond (1999) for samples from each of the six soil classes (Fig. 1), compared favorably with air-water retention curves predicted using the Haverkamp and Parlange method. The air entry pressure for each airwater retention curve was scaled to the organic-water system using a ratio of interfacial tensions. The Brooks-Corey (1964) extension of the Burdine (1953) model was employed to define a relative permeability function. Hysteresis due to

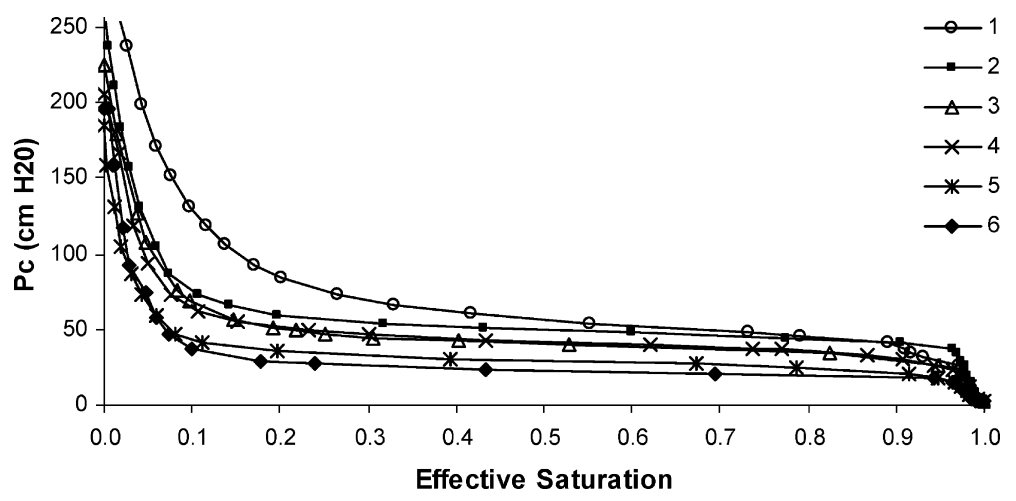

Fig. 1. Measured air/water retention curves for soil classes 1-6 
nonwetting phase entrapment was incorporated through the inclusion of apparent water saturations with the quantity of entrapped organic estimated using the method of Kaluarachchi and Parker (1992).

To explore the influence of spatial variability on organic retention, four distinct ensemble sets of 50 realizations were generated with alternative porosity, permeability, and $\mathrm{P}_{\mathrm{c}}$-Sat parameter assignments (Table 1). A conventional parameter estimation approach was used to create a reference set. In this set, random $d_{10}$ fields, conditioned to the $167 d_{10}$ measurements across the study area, were generated for each realization using Sequential Gaussian Simulation (SGS). A uniform average porosity of 0.36 was assumed and $k$ values were calculated using the Kozeny-Carman relationship (eq. 1). Vertical permeability was reduced uniformly by a factor of 0.5 to account for anisotropy related to aquifer stratification. The entry pressure from a single representative $\mathrm{P}_{c}$-Sat curve, estimated from a weighted average grain size distribution curve, was scaled to the permeability and porosity fields using Leverett (1941) scaling:

$P_{c 2}=P_{c 1} \sqrt{\frac{k_{1} \phi_{2}}{k_{2} \phi_{1}}}$.

Here, representative and scaled values are denoted with the subscripts 1 and 2, respectively.

In contrast to the reference set, $d_{10}$ fields for three additional simulation sets were generated using sequential indicator simulation (SIS) (Deutsch and Journel, 1998). Indicator classes were assigned based on the six identified soil classes (Lemke et al., 2002, accepted for publication). Three-dimensional indicator fields were then generated at a $0.3 \mathrm{~m}$ increment over a $24 \times 18 \times 9 \mathrm{~m}$ grid using conditional SIS. Nonuniform $d_{10}$ values were assigned to each grid node based on the simulated indicator value using a procedure described by Lemke et al. (2002). A uniform porosity of 0.36 was assumed for the first simulation set, whereas porosity for the second and third sets was estimated as an independent random field using SGS. Permeability was subsequently estimated from the $\phi$ and $d_{10}$ values at each node using Eq. (1). Leverett scaling (Eq. 4) of $P_{b}$ from a single representative Pc-Sat curve was used in the first two simulation sets. For simulation set $3, P_{b}$ and $\lambda$ values were estimated using the Haverkamp and Parlange (1986) method applied to weighted grain size distributions corresponding to the simulated indicator class and porosity at each grid node.

Table 1. Variable treatment of intrinsic permeability, porosity, and Brooks-Corey capillary pressure-saturation parameters among the four alternative simulation sets considered

\begin{tabular}{|c|c|c|c|c|}
\hline & $\begin{array}{l}\text { Reference set } \\
\text { (Conventional) }\end{array}$ & $\begin{array}{l}\text { Simulation } \\
\text { Set } 1\end{array}$ & Simulation Set 2 & Simulation Set 3 \\
\hline $\begin{array}{l}\text { Permeability, } \\
k\end{array}$ & SGS & $\begin{array}{l}\text { SIS } \\
k=f\left(d_{10}, \phi\right)\end{array}$ & SIS $k=f\left(d_{10}, \phi\right)$ & SIS $k=f\left(d_{10}, \phi\right)$ \\
\hline Porosity, $\phi$ & Uniform & Uniform & Variable (SGS) & Variable (SGS) \\
\hline$P_{c}$-Sat & Uniform $\lambda$, & Uniform $\lambda$, & Uniform $\lambda$, & $\begin{array}{l}\text { Haverkamp } \\
\text { and Parlange: }\end{array}$ \\
\hline (Brooks-Corey) & $\begin{array}{l}\text { scaling of } \\
\text { single } P_{b}\end{array}$ & $\begin{array}{l}\text { scaling of } \\
\text { single } P_{b}\end{array}$ & scaling of single $P_{b}$ & $\begin{array}{l}\lambda, P_{b}=f(\text { SIS } \\
\text { class, } \phi)\end{array}$ \\
\hline Leverett scaling & $P_{b}=f(\sqrt{k})$ & $P_{b}=f(\sqrt{k})$ & $P_{b}=f\left(\sqrt{k=f\left(d_{10}, \phi\right)}\right)$ & None \\
\hline
\end{tabular}




\section{Flow and transport simulation}

The influence of the four alternative spatial variability models on predictions of DNAPL entrapment and recovery was investigated using three procedures. First, MVALOR was used to simulate the infiltration of PCE in $7.9 \times 9.8 \mathrm{~m}$ twodimensional profiles extracted from each simulation set (Lemke et al., 2002). Horizontal and vertical grid discretization was 30.48 and $7.62 \mathrm{~cm}$, respectively. The simulated spill involved the release of $961(156 \mathrm{~kg})$ of PCE over four grid cells at the top of the model domain at a constant flux of $0.24 \mathrm{l}^{\mathrm{d}}$ day $^{-1}$ for 400 days, with an additional 330 days for subsequent organic infiltration and redistribution. An ensemble of 50 realizations was examined from each set.

Second, MVALOR PCE saturation distributions from eight realizations (two from each set) were used as initial conditions for the independent simulation of organic recovery under SEAR, water flood, and natural gradient conditions using MISER. Constant pressure boundary conditions were applied to simulate PCE dissolution under natural gradient (0.001) conditions for a period of 1000 days. The water flood simulations involved injection of approximately five pore volumes at a higher gradient $(0.130)$ over a period of 6 days. Both natural gradient and water flood simulations utilized the PCE solubilization mass transfer correlation of Powers et al. (1992). Simulated SEAR involved the injection of approximately 1.5 pore volumes of $4 \%$ (by volume) polyoxyethylene (20) sorbitan monooleate (Tween 80 ) aqueous surfactant solution over two days followed by approximately 3.5 pore volumes of water over four days. SEAR simulations utilized the rate limited PCE solubilization mass transfer correlation of Taylor et al. (2001) following the approach of Rathfelder et al. (2001). At equilibrium, the PCE solubilization capacity of $4 \%$ Tween 80 is approximately $26880 \mathrm{mg} \cdot \mathrm{l}^{-1}$. The longitudinal dispersivity value was set to $0.3 \mathrm{~m}$ based on history matching of alcohol breakthrough curve behavior in three-dimensional solute transport models (Drummond et al., 2000). A transverse dispersivity value of $0.0075 \mathrm{~m}$ was chosen after sensitivity to values ranging from 0.0075 to $0.06 \mathrm{~m}$ demonstrated a negligible influence on PCE recovery and aqueous phase mass flux estimates. Organic sorption was neglected after the influence of linear, equilibrium PCE sorption on post-SEAR PCE effluent concentrations was determined to be small.

Third, an alternative SEAR scenario was simulated for two realizations from each set. This approach combined natural gradient, surfactant injection and water flood in four stages. Initially, natural gradient flow was simulated for 100 days. This period was sufficient to pass more than one pore volume of water through the model domain and stabilize PCE effluent concentrations. Next, $4 \%$ Tween 80 SEAR was simulated at a higher gradient $(0.130)$ for two days followed by water flood at the same gradient for 10 days. Finally, natural gradient flow was resumed for 100 days. This approach allows pre- and post-SEAR PCE effluent concentrations to be compared under natural gradient conditions. As an initial investigation into predicted contaminant mass flux as a function of PCE removal, the duration of the surfactant injection period was varied from zero (i.e. water flood only) to 25 days in order to generate a series of mass flux estimates associated with modeled remediation efficiencies ranging from 0 to 99\% DNAPL recovery.

\section{4}

\section{Results}

Figure 2 illustrates the initial PCE distribution for a single realization from the reference simulation set, along with residual PCE distributions following independently simulated water flooding and SEAR. Similar results are presented for 


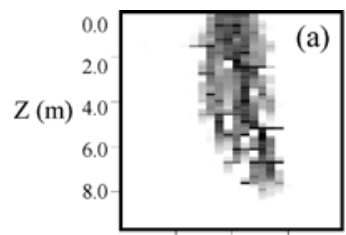

$\mathrm{X}(\mathrm{m})$
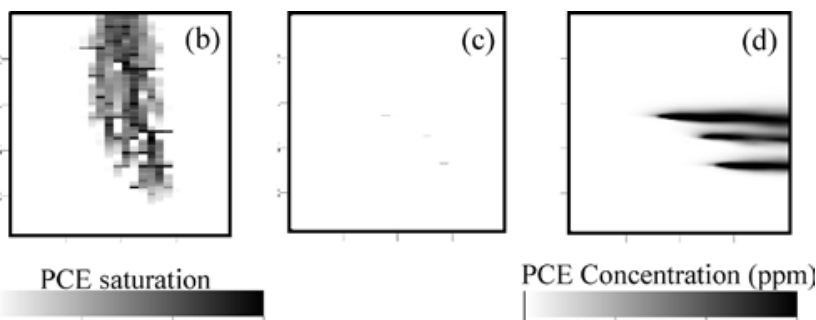

$0.0 \quad 50 \quad 100 \quad 150$

Fig. 2. Simulated PCE distributions for Reference Simulation Set, realization 1: a initial NAPL saturation, $\mathbf{b}$ post-water flood NAPL saturation, $\mathbf{c}$ post-SEAR NAPL saturation, d post-SEAR aqueous PCE concentration. The maximum value of the PCE saturation color scale is set to $3 \%$ to highlight spatial variability in low saturation values
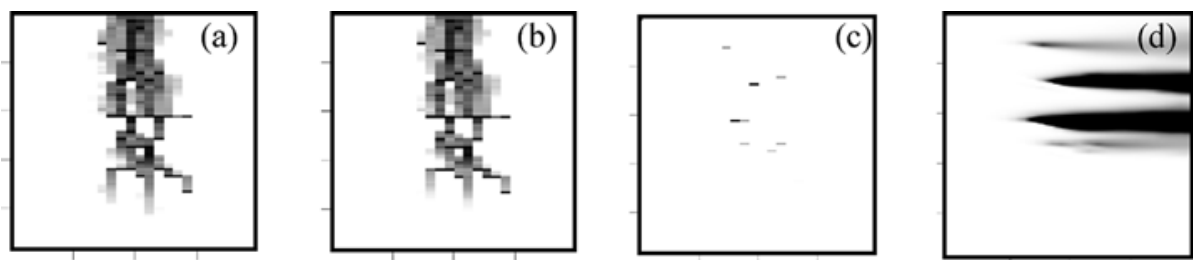

Fig. 3. Simulated PCE distributions for Simulation Set 1, realization 1: a initial NAPL saturation, $\mathbf{b}$ post-water flood NAPL saturation, $\mathbf{c}$ post-SEAR NAPL saturation, $\mathbf{d}$ postSEAR aqueous PCE concentration. Scale bars are presented in Fig. 2
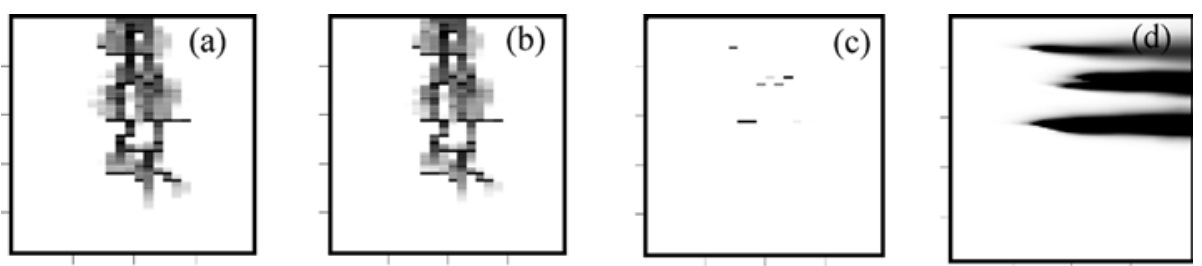

Fig. 4. Simulated PCE distributions for Simulation Set 2, realization 1: a initial NAPL saturation, $\mathbf{b}$ post-water flood NAPL saturation, $\mathbf{c}$ post-SEAR NAPL saturation, $\mathbf{d}$ postSEAR aqueous PCE concentration. Scale bars are presented in Fig. 2
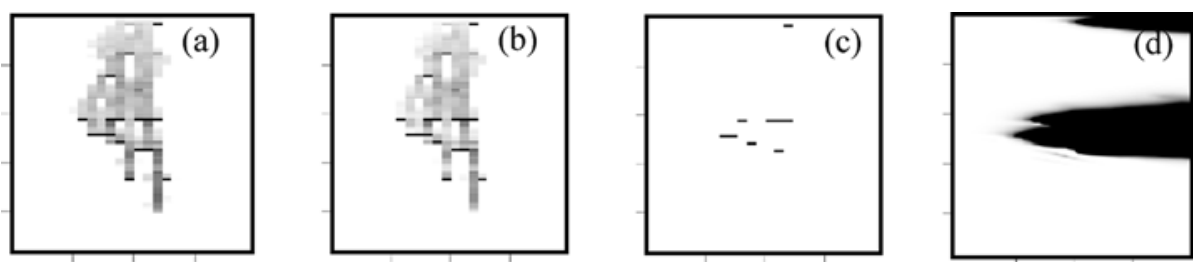

Fig. 5. Simulated PCE distributions for Simulation Set 3, realization 1: a initial NAPL saturation, $\mathbf{b}$ post-water flood NAPL saturation, $\mathbf{c}$ post-SEAR NAPL saturation, $\mathbf{d}$ postSEAR aqueous PCE concentration. Scale bars are presented in Fig. 2

individual realizations from sets 1, 2 and 3 in Figs. 3, 4 and 5, respectively. For plotting purposes, the maximum value of the gray scale is set to $3 \%$ to highlight spatial variability in low saturation values. In each realization, however, high organic saturations were predicted by MVALOR in a small number of cells. Maximum saturations ranged from 17 to $37 \%$ in the reference and simulation 
sets 1 and 2, and 90 to $92 \%$ in simulation set 3. The presence of PCE in these cells, visible in Figs. $2 c-5 c$, persists following simulated SEAR. PCE dissolution from these cells is responsible for post-SEAR aqueous phase contaminant mass flux. Both the magnitude and distribution of simulated dissolved phase contaminant flux are a function of the number and spatial arrangement of these cells.

Table 2 summarizes results for independently simulated natural gradient, water flood, and SEAR for each of the realizations presented in Figs. 2-5. Effluent PCE concentrations reported are flux-weighted averages over the right hand boundary of the model domain. Post-SEAR concentrations depicted in Figs. 2d$5 \mathrm{~d}$ and reported in Table 2 were calculated under water flood gradient conditions. Figure 6 depicts the change in PCE concentration with time for one realization from each set during a 2-day surfactant/4-day water flood simulation. Initial concentrations are representative of flow under natural gradient conditions. Enhanced solubility of PCE during the surfactant flush is reflected in peak concentrations during the first two days. Concentrations during the subsequent water flood (days 2-6) began to equilibrate at values approximately one to two orders of magnitude below the initial natural gradient concentrations.

Results from the initial investigation of predicted contaminant mass flux as a function of remediation efficiency are presented in Fig. 7. Flux-weighted PCE concentrations at the right hand model boundary are plotted as a function of \% DNAPL removed from the model domain. Concentrations plotted in Fig. 7 reflect 100 days of post-remediation flow under natural gradient flow conditions. Two

Table 2. MISER simulation results for a single realization from each simulation set

\begin{tabular}{lccc}
\hline & $\begin{array}{c}\text { Natural } \\
\text { gradient }\end{array}$ & Water flood & SEAR \\
& \multicolumn{1}{c}{} & \\
\hline Simulation duration (days) & 1000 & 6 & 6 \\
Reference case & & & \\
Pore volumes & 6.26 & 4.94 & $1.66+3.34^{*}$ \\
PCE mass removed (kg) & 19.55 & 14.56 & 156.76 \\
\% PCE removal & 12.40 & 9.27 & 99.79 \\
Effluent PCE concentration (ppm) & 113.112 & 108.363 & 0.232 \\
PCE mass flux (kg day ${ }^{-1}$ ) & 0.0197 & 2.4841 & 0.0054 \\
Simulation 1 & & & \\
Pore volumes & 6.19 & 4.89 & $1.64+3.30^{*}$ \\
PCE mass removed (kg) & 18.31 & 14.29 & 155.67 \\
\% PCE removal & 11.67 & 8.31 & 99.23 \\
Effluent PCE concentration (ppm) & 106.247 & 98.589 & 0.528 \\
PCE mass flux (kg day ${ }^{-1}$ ) & 0.0183 & 2.2352 & 0.0121 \\
Simulation 2 & & & \\
Pore volumes & 6.44 & 5.08 & $1.71+3.43^{*}$ \\
PCE mass removed (kg) & 18.87 & 13.31 & 155.28 \\
\% PCE removal & 12.03 & 8.49 & 99.00 \\
Effluent PCE concentration (ppm) & 105.662 & 96.280 & 0.683 \\
PCE mass flux (kg day ${ }^{-1}$ ) & 0.0192 & 2.2989 & 0.0166 \\
Simulation 3 & & & \\
Pore volumes & 6.47 & 5.11 & $1.71+3.42^{*}$ \\
PCE mass removed (kg) & 19.39 & 13.23 & 93.26 \\
\% PCE removal & 12.36 & 8.44 & 59.47 \\
Effluent PCE concentration (ppm) & 106.904 & 93.987 & 3.048 \\
PCE mass flux (kg day ${ }^{-1}$ ) & 0.0195 & 2.2547 & 0.0735 \\
\hline
\end{tabular}

${ }^{*}$ Pore volumes surfactant solution + pore volumes water 


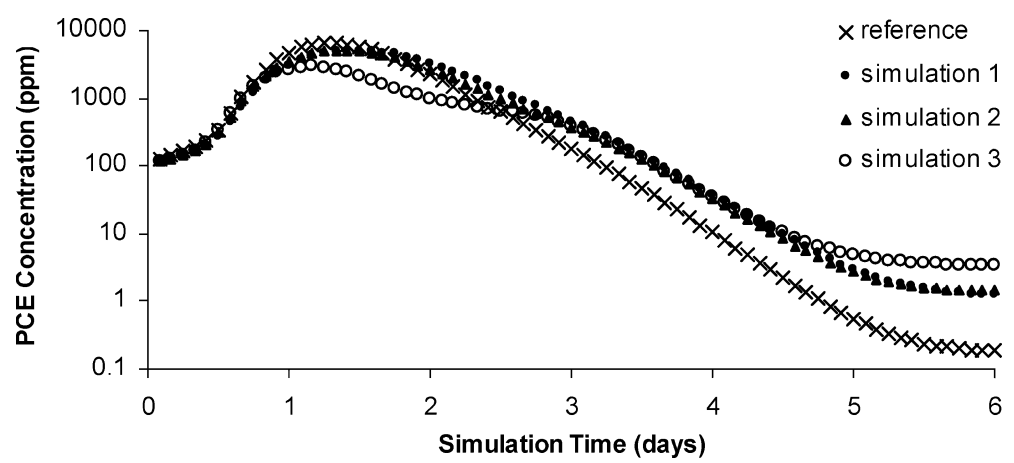

Fig. 6. Flux-averaged aqueous PCE concentration at downstream boundary during two-day surfactant flush followed by a four-day water flood. Results of a single realization from the reference set and each of the three alternative simulation sets are included. PCE concentrations at time zero reflect contaminant mass flux under natural gradient conditions. PCE concentrations at day 6 reflect water flood gradient conditions

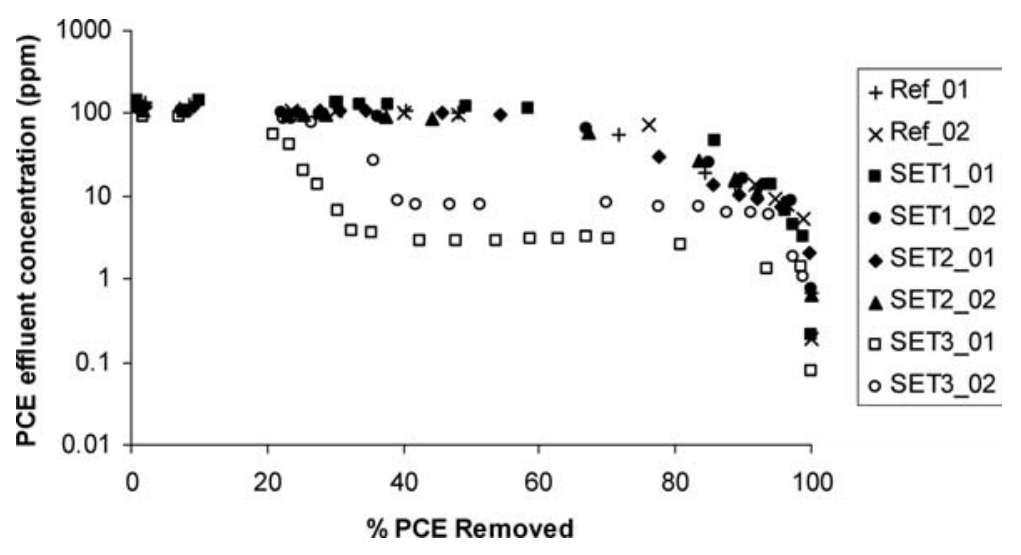

Fig. 7. Flux-averaged aqueous PCE concentration at the downstream model boundary following surfactant flush plotted as a function of \% PCE removed. Two realizations each from the reference set and simulation sets 1 to 3 are included. Simulations from realizations incorporating Leverett scaling (closed symbols) follow a similar path that contrasts with paths of simulations employing independent $k$ and $\mathrm{P}_{c}$ relations (open symbols)

realizations from each simulation set were analyzed. The six realizations incorporating Leverett scaling (reference plus sets 1 and 2) display similar patterns of [PCE] vs. \% DNAPL removed. In these realizations, removal of 70-80\% of DNAPL was required before dissolved phase PCE concentrations began to fall appreciably. The two realizations incorporating uncorrelated $\mathrm{P}_{\mathrm{c}}$-Sat and $k$ fields (set 3) display more variability, but predict PCE concentration reductions between one to two orders of magnitude after only 40-60\% DNAPL removal. This favorable behavior results from the initial simulated infiltration and entrapment of PCE in a small number of highly saturated cells or pools. Such DNAPL source zone architecture was observed only in realizations that did not directly scale entry pressures to permeability (set 3). In these realizations, PCE in the remaining cells was generally at very low residual saturations and was removed early in the remediation process, leaving only isolated pockets of high organic saturation cells as a persistent source of dissolved contaminant. After DNAPL removal in excess of 98\%, 
the configuration and saturations of the few remaining cells containing organic was similar in all simulation sets. DNAPL removal in excess of $99 \%$ of the original mass in place was then necessary before further reductions in dissolved phase concentrations were predicted.

\section{5}

\section{Discussion and conclusions}

Simulation results presented here demonstrate that alternative models of spatial variability in aquifer parameters strongly influence DNAPL entrapment, even in a homogeneous, nonuniform sand aquifer. PCE saturation profiles from the reference case and simulation sets 1 and 2 (Figs. 2a, 3a and 4a) display irregular downward migration paths due to macroscopic permeability variations in the simulation grid of the type discussed by Kueper and Frind (1991). Independent simulation of a variable porosity field contributed to greater variability in the estimated $k$ field and distribution of PCE saturations in simulation set 2 relative to set 1 . Although realizations in simulation sets 2 and 3 share identical porosity and permeability distributions, correlation of $P_{b}$ with grain size classes and the assignment of $\mathrm{P}_{\mathrm{c}}$-Sat parameters independently of the $k$ field led to a greater spatial continuity of similar entry pressure values that accounts for the increased incidence of DNAPL pooling and higher maximum saturations observed in simulation set 3 (Lemke et al., 2002, accepted for publication).

Dissolution of pooled and residual PCE under natural gradient conditions resulted in flux-weighted concentrations on the order of $100 \mathrm{ppm}$ across the downstream model boundary (Table 2). Values below the equilibrium PCE solubility limit of $149 \mathrm{ppm}$ at $20{ }^{\circ} \mathrm{C}$ (Horvath, 1982, p. 501) are attributable to dilution from water flowing through uncontaminated portions of the profiles and dispersion during transport. Although laboratory experiments indicate that dissolved contaminant concentrations in water flowing through zones of residual organic saturation rise quickly to near equilibrium levels (Anderson et al., 1992), aqueous phase concentrations below the solubility limit are typical of field measurements made at sites contaminated by chlorinated solvents such as PCE (Feenstra and Guiguer, 1996).

Water flooding under the conditions simulated led to predicted recovery of 8 to $10 \%$ of entrapped PCE. Removal of this fraction of entrapped NAPL did not change the source zone configuration (compare Figs. 2-5(a) with 2-5(b)). Consequently, predicted post-water flood PCE effluent concentrations were not significantly reduced with respect to initial concentrations or natural gradient conditions. Dissolved phase contaminant mass flux was approximately two orders of magnitude larger under water flood conditions than natural gradient conditions, however, due to the two order of magnitude increase in the imposed water flood gradient.

SEAR simulations predict that approximately $99 \%$ of PCE can be removed by surfactant flushing for the selected realizations from the reference set and simulation sets 1 and 2. A similar surfactant flush produced approximately $60 \%$ PCE removal in simulation 3 due to a greater degree of pooling and higher entrapped DNAPL saturations. Nevertheless, all three simulations demonstrated an approximate two order of magnitude decrease in PCE mass flux relative to water flood conditions (Table 2) as well as a two order of magnitude reduction in PCE concentration relative to natural gradient conditions (Fig. 6).

Flux-weighted PCE concentrations predicted as a function of \% DNAPL removed differed for realizations with correlated and uncorrelated $\mathrm{P}_{\mathrm{c}}$-Sat and 
permeability fields. In the former case, removal of more than $70 \%$ of the DNAPL originally in place was required before appreciable decreases in aqueous phase organic concentrations were predicted. In the latter case, PCE concentration reductions between one to two orders of magnitude were predicted with as little as $40 \%$ DNAPL removal. Variability in the initial results presented here must be confirmed through analysis of an ensemble of realizations from each simulation set before we can confidently conclude that differences in observed model behavior are statistically significant.

It is likely that similar differences between correlated and uncorrelated system behavior would be observed in aquifers characterized by discretely heterogeneous properties or greater variance in $\ln (K)$ than the nonuniform aquifer studied here. Other modelers simulating the effects of DNAPL spills using correlated $\mathrm{P}_{\mathrm{c}}$-Sat and $k$ fields (generated with Gaussian stochastic algorithms and Leveret scaling) have found that spreading and infiltration behavior is influenced by spatial variability. For example, Dekker and Abriola (2000) found that increased variance in $\ln (\mathrm{K})$ led to increased horizontal spreading and higher maximum organic saturations. Variability in DNAPL infiltration and saturation metrics in response to different geostatistical correlation lengths and organic spill sizes also has been demonstrated (Kueper and Gerhard, 1995; Dekker and Abriola, 2000). For aquifer models incorporating uncorrelated $\mathrm{P}_{c}$-Sat and $k$ fields, enhanced differences in DNAPL infiltration and entrapment in response to greater aquifer spatial variability could enhance the differences in DNAPL recovery behavior described in this study.

The results of the set of numerical experiments presented here suggest that (1) independent variation in more than one aquifer parameter can increase the variance of predicted DNAPL saturation distributions, (2) assignment of Pc-Sat parameters based on spatially correlated grain size classes can have a significant influence on predicted entrapped DNAPL distributions, and (3) evolving configurations of the resultant entrapped organic phase liquids during source zone remediation can contribute to discernable variability in recovery efficiency and the response of dissolved contaminant mass flux to the percentage of DNAPL recovered. For the example realizations shown, removal of $60 \%$ to $99 \%$ of entrapped PCE reduced dissolved contaminant concentration and mass flux by two orders of magnitude relative to natural gradient conditions.

\section{References}

Abriola LM, Drummond CD, Lemke LD, Rathfelder KM, Pennell KD, Petrovskis EK, Daniels G (2001) Surfactant enhanced aquifer remediation: application of mathematical models in the design and evaluation of a pilot-scale test. In: Thornton SF, Oswald SE, (eds), Groundwater Quality 2001, pp. 303-309. Sheffield, UK

Abriola LM, Lang J, Rathfelder K (1997) Michigan soil-vapor extraction remediation (MISER) model - A computer program to model bioventing of organic chemicals in unsaturated geological material. EPA/600R-97/099, U. S. Environmental Protection Agency Abriola LM, Rathfelder KM, Yadav S, Maiza M (1992) VALOR code version 1.0: a PC code for simulating subsurface immiscible contaminant transport. EPRI TR-101018, Electric Power Research Institute

Bear J (1972) Dynamics of Fluids in Porous Media. Elsevier, New York

Brooks RH, Corey AT (1964) Hydraulic properties of porous media. Colorado State University, Fort Collins

Burdine NT (1953) Relative permeability calculations from pore size distribution data. AIME Petroleum Trans. 198: 71-78

Dekker TJ, Abriola LM (2000) The influence of field scale heterogeneity on the infiltration and entrapment of dense nonaqueous phase liquids in saturated formations. J. Contaminant Hydrol. 42: 187-218 
Deutsch CV, Journel AG (1998) GSLIB Geostatistical Software Library and User's Guide, second edition. Oxford University Press, New York

Drummond CD, Lemke LD, Rathfelder KM, Hahn EJ, Abriola LM (2000) Simulation of surfactant-enhanced PCE recovery at a pilot test field site. In: Wickramanayake GB, Gavaskar AR, Gupta N, (eds), Treating Dense Nonaqueous Phase Liquids (DNAPLs): Remediation of Chlorinated and Recalcitrant Compounds, pp. 77-84 Batelle Press, Columbus, $\mathrm{OH}$, Monterey, CA

Haverkamp R, Parlange JY (1986) Predicting the water-retention curve from particle-size distribution: 1. Sandy soils without organic matter. Soil Science 142: 325-339

Kaluarachchi JJ, Parker JC (1992) Multiphase flow with a simplified model for oil entrapment. Transport in Porous Media 7: 1-14

Kueper BH, Frind EO (1991) Two-phase flow in heterogeneous porous media 1. Model development. Water Resour. Res. 27: 1049-1057

Kueper BH, Gerhard JI (1995) Variability of point source infiltration rates for two-phase flow in heterogeneous porous media. Water Resour. Res. 31: 2971-2980

Lemke LD, Abriola LM, Goovaerts P (2002) Exploration of the influence of hydraulic property correlation on predictions of DNAPL infiltration and entrapment. In: Findikakis AN, (ed.), Bridging the Gap between Measurements and Modeling in Heterogeneous Media, pp. 58-62, IAHR, Berkeley, California

Lemke LD, Abriola LM, Goovaerts P (2003) DNAPL source zone characterization: influence of hydraulic property correlation on predictions of DNAPL infiltration and entrapment. Water Resour. Res.

Lemke LD, Hahn EJ, Drummond CD, Rathfelder KM, Abriola LM, Goovaerts P (2000) Comparison of sequential Gaussian and sequential indicator geostatistical simulations using three-dimensional flow and transport models in a homogeneous, non-uniform aquifer [abstr.]. In: North American Council on Geostatistics, Ann Arbor, MI

Leverett MC (1941) Capillary behavior in porous solids. American Institute of Mining and Metallurgical Engineers Transactions, Petroleum Development and Technology 142: 152-169

Powers SE, Abriola LM, Weber WJ, Jr. (1992) An experimental investigation of nonaqueous phase liquid dissolution in saturated subsurface systems: steady state mass transfer rates. Water Resour. Res. 28: 2691-2705

Rathfelder KM, Abriola LM (1998) Influence of capillarity in numerical modeling of organic liquid redistribution in two-phase systems. Adv. Water Resour. 21: 159-170

Rathfelder KM, Abriola LM, Taylor TP, Pennell KD (2001) Surfactant enhanced recovery of tetrachloroethylene from a porous medium containing low permeability lenses 2 . Numerical simulation. Journal of Contaminant Hydrology 48: 351-374

Salehzadeh A, Demond AH (1999) Pressure cell for measuring capillary pressure relationships of contaminated sands. J. Environ. Eng. 125: 385-388

Schad H (1993) Geostatistical analysis of hydraulic conductivity related data based on core samples from a heterogeneous fluvial aquifer. In: Vincenzo C, Giovanni G, Donato P, (eds), Statistics of Spatial Processes: Theory and Applications, pp. 205-212, Bari, Italy Taylor TP, Pennell KD, Abriola LM, Dane JH (2001) Surfactant enhanced recovery of tetrachloroethylene from a porous medium containing low permeability lenses. 1. Experimental studies. J. Contaminant Hydrol. 48: 325-350

Woodbury AD, Sudicky EA (1991) The geostatistical characterization of the Borden aquifer. Water Resour. Res. 27: 533-546 